\title{
Velocity-Dependent Friction and Diffusion for Grains in Neutral Gases, Dusty Plasmas and Active Systems
}

\author{
S.A. Trigger ${ }^{1}$, G.J.F.van Heijst ${ }^{2}$, P.P.J.M. Schram ${ }^{2}$ \\ 1 Joint Institute for High Temperatures, Russian Academy of Sciences, 13/19, Izhorskaia Str., \\ Moscow127412, Russia. email: satrig@freenet.de \\ 2 Eindhoven University of Technology, P.O. Box 513, MB 5600 Eindhoven, The Netherlands
}

\begin{abstract}
A self-consistent and universal description of friction and diffusion for Brownian particles (grains) in different systems, as a gas with Boltzmann collisions, dusty plasma with ion absorption by grains, and for active particles (e.g., cells in biological systems) is suggested on the basis of the appropriate Fokker-Planck equation. Restrictions for application of the Fokker-Planck equation to the problem of velocity-dependent friction and diffusion coefficients are found. General description for this coefficient is formulated on the basis of master equation. Relation of the diffusion coefficient in the coordinate and velocity spaces is found for active (capable to transfer momentum to the ambient media) and passive particles in the framework of the Fokker-Planck equation.

The problem of anomalous space diffusion is formulated on the basis of the appropriate probability transition (PT) function. The method of partial differentiation is avoided to construct the correct probability distributions for arbitrary distances, what is important for applications to different stochastic problems. Generale equation for time-dependent PT function is formulated and discussed.

Generalized friction in the velocity space is determined and applied to describe the friction force itself as well as the drag force in the case of a non-zero driven ion velocity in plasmas. The negative friction due to ion scattering on grains exists and can be realized for the appropriate experimental conditions.
\end{abstract}

PACS: 52.27.Lw, 52.20.Hv, 05.40.-a, 05.40.Fb 


\section{INTRODUCTION}

Interest in Brownian dynamics is conditioned by a large variety of applications: granular systems, including dusty plasmas, various objects in biological systems, physical-chemical systems, et cetera. For open systems and for systems with non-elastic processes the relations between the friction and diffusion coefficients and even the correct specific forms of the Fokker-Planck equation are not still established. Recently the friction and diffusion

coefficients as functions of the grain velocity $V$ were derived for dusty plasmas [1]. Due to ion absorption by the grains, the friction coefficient can become negative [2] for the simplest model of dusty plasmas with ion absorption without atom regeneration on the grain's surface. This implies that the problem of negative friction has to be considered for more complicated models and realistic situations in dusty plasmas, in particular taking into account the grain's mass conservation for, which is essential for large times of the process, in spite of big difference between ion and grain masses. In the present paper we consider the specific forms of the probability transition (PT) [3] for Boltzmann-type and and nonBoltzmann-type of collisions to calculate and compare the velocity dependent friction and diffusion coefficients in different systems, including gases, dusty plasmas and active particles. Our main goal in this paper is determination of the velocity-dependent friction and diffusion coefficients for arbitrary grain velocity and consideration of diffusion in the coordinate and velocity spaces for different systems and conditions.

In Section 2 the Fokker-Planck equation and self-consistent description of velocitydependent friction and diffusion are discussed in details on basis of the PT function (PT). In Section 3 we consider in detail the probability transition function for Boltzmann-type collisions, in particular, for the Boltzmann's spheres. Friction and diffusion coefficients are calculated on the basis of the Fokker-Planck equation. The restriction for application of the Fokker-Planck equation to the fast motion of grains for the Boltzmann's type of collisions and necessity to use the appropriate master equation with prescribed PT function is shown. The general formulae for the velocity-dependent friction and diffusion coefficients in the velocity space are established on the basis of master equation. In Section 4 the absorption collisions for ions by grains is done for dusty plasmas with a high grain charge. In the framework of the simplest model with a fixed mass of grains the phenomena of negative friction is found. The friction coefficient changes sign for some value of the grain velocity. Manifestation of negative friction due to ion absorption in dusty plasmas is the consequence of the model. Opportunity to realize this process in the experiment can be considered on more elaborated model with grain mass conservation. In Section 5 we consider diffusion in the coordinate space on the basis of the Fokker-Planck kinetic equation. The general relation between the diffusion coefficient in the coordinate space and velocity-dependent diffusion and friction coefficients in the velocity space are found. Relation of these results with the known limiting cases is established. The results are applied to the self-motion of grains (e.g. cells) in biological systems. In Section 6 the different types of anomalous diffusion in the coordinate space are considered on the basis of the appropriate master equation. As particular case we describes the Levy-flights, which are very important for many applications. We also formulated the generalized diffusion equation for the time-dependent PT function in the coordinate space, investigated it for some particular cases and suggest to apply for subdiffusion processes. In Section 7 we shortly describe the generalized friction, which appears when 
some other vectors, except grain velocity, are essential to determination of PT function in the velocity space. The application of such consideration is done in Section 8, where the ion flow with some driven velocity (which is the particular case of such additional vector in PT function) scatters on grains. Due to this process the total friction can be negative for small grain velocity and provides the mechanism for acceleration and heating grains in dusty plasmas. The conclusions are presented in the Section 9.

\section{FOKKER-PLANCK EQUATION AND SELF CONSISTENT VELOCITY-DEPENDENT FRICTION AND DIFFUSION}

The friction and diffusion coefficients $\beta(V), D_{\|}(V)$ and $D_{\perp}(V)$ on the basis of the FokkerPlanck equation are determined via PT function $w(\mathbf{P}, \mathbf{q})[3]$ as

$$
\begin{gathered}
\beta(V)=\frac{1}{P^{2}} \int d^{s} \mathbf{q}(\mathbf{P} \mathbf{q}) w(\mathbf{P}, \mathbf{q}), \\
D_{\|}(V)=\left(1 / 2 M^{2} P^{2}\right) \int d^{s} \mathbf{q}(\mathbf{P} \mathbf{q})^{2} w(\mathbf{P}, \mathbf{q}) .
\end{gathered}
$$

and

$$
D_{\perp}(V)=\frac{1}{2(s-1) M^{2} P^{2}} \int d^{s} \mathbf{q}\left[P^{2} q^{2}-(\mathbf{P q})^{2}\right] w(\mathbf{P}, \mathbf{q}) .
$$

Here $(\mathbf{P q})$ is the scalar product in velocity space of the dimension $s$ and $\mathbf{P} \equiv M \mathbf{V}$, where $M$ and $V$ are respectively the mass and velocity of the grain.

We determine the $\mathrm{PT}$ function $w(\mathbf{P}, \mathbf{q}) \equiv w\left(\mathbf{P}, \mathbf{P}^{\prime}-\mathbf{P}\right)\left(\mathbf{q} \equiv \mathbf{P}^{\prime}-\mathbf{P}\right.$ represents the momentum transfer for the considered process, e.g. collision) in the same way (convenient for consideration of the Fokker-Planck equation) as was done in [5]:

$$
\begin{array}{r}
\frac{d f_{g}(\mathbf{P}, t)}{d t}=I_{g}(\mathbf{P}, t)=\int d \mathbf{q}\left\{w(\mathbf{P}+\mathbf{q}, \mathbf{q}) f_{g}(\mathbf{P}+\mathbf{q}, \mathbf{t})\right. \\
\left.-w(\mathbf{P}, \mathbf{q}) f_{g}(\mathbf{P}, t)\right\},
\end{array}
$$

The corresponding to Eq. (4) Fokker-Planck equation can be written as:

$$
\frac{d f_{g}(\mathbf{P}, t)}{d t}=\frac{\partial}{\partial P_{i}}\left[P_{i} \beta(\mathbf{P}) f_{g}(\mathbf{P})+M^{2} \frac{\partial}{\partial P_{j}}\left(D_{i j}(\mathbf{P}) f_{g}(\mathbf{P})\right)\right]
$$

The PT function $w(\mathbf{P}, \mathbf{q})$ is directly connected with the probability transition $w_{0}\left(\mathbf{P}, \mathbf{P}^{\prime}\right)$ in the Chapman-Kolmogorov equation (master equation, [4]) $w_{0}\left(\mathbf{P}^{\prime}, \mathbf{P}\right)=w\left(\mathbf{P}, \mathbf{P}-\mathbf{P}^{\prime}\right) \equiv$ $w(\mathbf{P}, \mathbf{q})$ :

$$
\frac{d f_{g}(\mathbf{P}, t)}{d t}=\int d \mathbf{P}^{\prime}\left\{w_{0}\left(\mathbf{P}, \mathbf{P}^{\prime}\right) f_{g}\left(\mathbf{P}^{\prime}, \mathbf{t}\right)-w_{0}\left(\mathbf{P}^{\prime}, \mathbf{P}\right) f_{g}(\mathbf{P}, t)\right\}
$$

It follows from Eqs. (1), (2) that the friction and diffusion coefficients always have to be calculated self-consistently. For the Boltzmann-type collisions between the light particles 
(conventionally atoms) and grains with masses $m$ and $M(m \ll M)$, respectively, the PT function $w(\mathbf{P}, \mathbf{q})$ and the coefficients $\beta(V)$ and $D(V)$ can be found for arbitrary cross-section (as will be done in Section 2). In Section 2 we also investigate the applicability of the Einstein relation to the Boltzmann-type collisions on the basis of the Fokker-Planck equation with velocity-dependent coefficients.

For some other type of collisions the problem of a single-valued form of $w(\mathbf{P}, \mathbf{q})$ exists, because the exact kinetic equation for such collisions is not known in many cases. The Einstein relation between friction and diffusion of grains can be easily violated for such type of non-Boltzmann collisions, even for very low grain velocity.

The solution of the stationary Fokker-Planck equation for the isotropic distribution function of the particles can be written [3] as:

$$
f_{g}(V)=\frac{C}{D_{\|}(V)} \exp \left[-\int_{0}^{V} d v v \frac{\beta^{*}(v)}{D_{\|}(v)}\right],
$$

where $C$ is a constant, providing normalization and $D_{\|}$is the longitudinal part of the diffusion tensor:

$$
D_{i j}(\mathbf{P})=\frac{P_{i} P_{j}}{P^{2}} D_{\|}(P)+\left(\delta_{i j}-\frac{P_{i} P_{j}}{P^{2}}\right) D_{\perp}(P) .
$$

The function $\beta^{*}(V)$ is related with $\beta(V)$ by the equality:

$$
\beta^{*}(V)=\beta(V)+\frac{s-1}{V^{2}}\left(D_{\|}(V)-D_{\perp}(V)\right) .
$$

From Eq. (7) follows:

$$
\beta^{*}(V)=-\frac{1}{V} \frac{\partial D_{\|}}{\partial V}-\frac{D_{\|}}{V f_{g}} \frac{\partial f_{g}}{\partial V}
$$

Here the function $f_{g}$ is the stationary grain distribution function.

\section{BOLTZMANN-TYPE COLLISIONS: LIMITATIONS OF THE FOKKER-PLANCK EQUATION. MASTER EQUATION APPROACH}

For the Boltzmann-type collisions the stationary distribution $f_{g}(V)$ is a Maxwellian equilibrium $f_{g 0}(V)$ and Eq. (10) has the form

$$
\beta(V)=-\frac{1}{V} \frac{\partial D_{\|}(V)}{\partial V}+\frac{M D_{\|}(V)}{T},
$$

If $D$ is a function of $V$ the Einstein relation $\beta=M D / T$ is violated if the term with derivative in Eq. (11) is not small with respect to the parameter $m / M$. Only in that special case deviation from the Einstein relation in Eq. (11) can be neglected, according to the general conditions of applicability for the Fokker-Planck equation. For Boltzmann collisions it is possible to show that the PT function $w_{B}(\mathbf{P}, \mathbf{q})$ (with $\mathbf{P} \equiv \mathbf{P}_{\mathbf{a}}=M \mathbf{V}$ ) can be represented as: 


$$
w_{B}(\mathbf{P}, \mathbf{q})=\frac{1}{\mu^{2}} \int d \mathbf{u} \delta\left(\mathbf{u q}-\frac{q^{2}}{2 \mu}\right) \cdot \frac{d \sigma}{d o}\left[\arccos \left(1-\frac{q^{2}}{2 \mu^{2} u^{2}}\right), u\right] f_{b}\left(\mathbf{u}+\mathbf{V}-\mathbf{v}_{\mathbf{0}}\right) .
$$

Here $\mu$ is the reduced mass of the particles of species $a$ and $b$ (with masses $M$ and $m$, respectively), $d \sigma / d o$ is the differential cross-section for Boltzmann-type scattering and $f_{b}$ is the velocity distribution function for the light particles, which we assume to be Maxwellian with, for generality, some shifted velocity. It means small particles can have some driven velocity $\mathbf{v}_{\mathbf{0}}$.

For the case of hard spheres, when $d \sigma / d o=a^{2} / 4$ and the distribution function of the small particles $f_{b}=f_{b 0}$ is Maxwellian , Eq. (12) can be written as:

$$
w_{B}^{0}(\mathbf{P}, \mathbf{q})=\frac{a^{2} n_{b}}{4 q \mu^{2}}\left(\frac{m_{b}}{2 \pi T_{b}}\right)^{1 / 2} \exp \left[-\frac{m_{b}}{2 T}\left(\frac{\mathbf{V q}}{q}-\frac{q}{2 \mu}\right)^{2}\right]
$$

where $a$ is the reduced radius of the colliding spheres. Let us calculate the friction and diffusion coefficients on the basis of general equations (1),(2) and the PT function (13) for hard spheres. It is easy to show that the friction coefficient can be represented via the function of parabolic cylinder $D_{-3}$ in the form:

$$
\beta(V)=\beta(0) I\left(\frac{V}{v_{T}}\right), I \equiv \frac{3}{2 \omega} \int_{-1}^{1} d x x \exp \left[-\frac{\omega^{2} x^{2}}{2}+\frac{\omega^{2} x}{4}\right] D_{-3}(-\omega x),
$$

where $\omega \equiv V / v_{T}$ and $v_{T}=\sqrt{T / m}$ is the thermal velocity of the small particles. The value of the friction coefficient for the grain in the hard spheres media for $V=0$ is equal:

$$
\beta(0)=8 \sqrt{2 \pi} \mu n a^{2} v_{T} / 3 M .
$$

Likewise for the friction coefficient we derive

$$
D(V)=D(0) J\left(\frac{V}{v_{T}}\right), J=\frac{9}{2} \int_{-1}^{1} d x x^{2} \exp \left[-\frac{\omega^{2} x^{2}}{2}+\frac{\omega^{2} x}{4}\right] D_{-4}(-\omega x) .
$$

For $V=0$ the coefficients $D(0)$ and $\beta(0)$ are related $(\mu \simeq m)$ by Einstein's law:

$$
D(0)=\mu T \beta(0) / m M
$$

For the opposite case of very high velocity $V \gg v_{T}$ the formal expressions for the coefficients $\beta$ and $D$, based on self-consistent relations (1),(2) and the PT function for Boltzmann-type collisions (12) are:

$$
\begin{gathered}
\beta\left(V \gg v_{T}\right)=\frac{\pi \mu}{M} a^{2} n V \\
D\left(V \gg v_{T}\right)=\beta\left(V \gg v_{T}\right) \frac{2 \mu V^{2}}{3 M}=\frac{2 \pi \mu^{2}}{3 M^{2}} a^{2} n V^{3}
\end{gathered}
$$

The similar expressions have been found in [3] (although here we corrected the numerical multipliers order of unit) and evidently violate the Einstein relation. As it follows from 
Eq. (13) the conditions of applicability of the Fokker-Planck equation cannot be restricted only by the usual inequality (e.g. [5]) $\mathbf{q} \ll \mathbf{P}$. To find the full limitations for transition from the Boltzmann equation to the Fokker-Planck equation, let us consider the conditions for expansion of the function $w(\mathbf{P}+\mathbf{q}, \mathbf{q})$ by $\mathbf{q}$ in Eq. (4) for the simple particular case described by Eq. (13):

$$
w_{B}^{0}(\mathbf{P}+\mathbf{q}, \mathbf{q})=w_{B}^{0}(\mathbf{P}, \mathbf{q}) \exp \left[-\frac{m_{b}}{2 T}\left(\frac{\mathbf{V q}}{q}-\frac{q}{2 \mu}\right) \frac{2 q}{M}-\frac{m_{b}}{2 T} \frac{q^{2}}{M^{2}}\right] .
$$

To obtain the Fokker-Planck equation from the master equation (4) we have to expand the exponent in Eq. (20). Therefore besides the general condition $\mathbf{q} \ll \mathbf{P}$ the inequalities $|\mathbf{V}| \ll v_{T}^{2} M / q$ and $q^{2} / T M \ll 1$ have to be fulfilled simultaneously. An even more stronger limitation for applicability of Fokker-Planck equation for Boltzmann-type collisions $|\mathrm{V}| \ll$ $T / q$ follows from the fact that the stationary state of the distribution function for grains (without external fields) is Maxwellian. This means that for any finite $q$ there is some limitation on the values $|\mathbf{V}|$, which can be described by the Fokker-Planck equation. In particular, according these inequalities the limit $T \rightarrow 0$ cannot be realized for the Boltzmanntype collisions on the basis of the Fokker-Planck approximation simultaneously with finite | V |-values. In other words, the expressions for the friction and diffusion coefficients (18), (19) for a Boltzmann gas for the velocities, which are not satisfied the inequalities, mentioned above, are in contradiction with Eq. (11).

Because the Fokker-Planck equation itself is not valid in Boltzmann gas for high velocities, Eqs. (5), (11) is not applicable and our aim is to find the friction and diffusion coefficients for arbitrary velocities on the basis of the Chapman-Kolmogorov equation (6). For this equation in the absence of the external fields the stationary distribution for grains is, as easy to see, the Maxwellian if the transfer probability function is calculated with the Maxwellian distribution of the light particles (13). Equation (6) permits to determine in a physically justified way the friction and diffusion coefficients for arbitrary particle velocities in the same form (1),(2) as it was found from the Fokker-Planck equation, in spite the equation itself is not valid anymore.

In order to show this let us integrate equation (11) with the multiplayer $P_{\alpha}$ and with the product $P_{\alpha} P_{\beta}$. Then we arrive to the equations for the momentum:

$$
\begin{gathered}
\frac{d\langle\mathbf{P}\rangle}{d t}=-\int d \mathbf{P} f_{g}(\mathbf{P}) \int d \mathbf{P}^{\prime} w_{0}\left(\mathbf{P}^{\prime}, \mathbf{P}\right)\left(\mathbf{P}-\mathbf{P}^{\prime}\right)= \\
-\int d \mathbf{P} f_{g}(P) \int d \mathbf{q} w(\mathbf{P}, \mathbf{q}) \mathbf{q} \equiv-\int d \mathbf{P} f_{g}(P) \mathbf{P} \beta(\mathbf{P}) .
\end{gathered}
$$

Here $\langle\mathbf{P}\rangle$ is the momentum, averaged by the distribution function $f_{g}$ and $\beta(\mathbf{P})$ is exactly the same as in Eq. (1). Equation for the averaged product $\left\langle\mathbf{P}_{\alpha} \mathbf{P}_{\beta}\right\rangle$ is:

$$
\begin{array}{r}
\frac{d\left\langle\mathbf{P}_{\alpha} \mathbf{P}_{\beta}\right\rangle}{d t}=-\int d \mathbf{P} f_{g}(\mathbf{P}) \int d \mathbf{P}^{\prime}\left(\mathbf{P}_{\alpha} \mathbf{P}_{\beta}-\mathbf{P}_{\alpha}^{\prime} \mathbf{P}_{\beta}^{\prime}\right) w_{0}\left(\mathbf{P}^{\prime}, \mathbf{P}\right) \equiv \\
\int d \mathbf{P} f_{g}(\mathbf{P})\left[2 \frac{P_{\alpha} P_{\beta}}{P^{2}}\left(M^{2} D_{\|}(P)-P^{2} \beta(P)\right)+2 M^{2}\left(\delta_{\alpha \beta}-\frac{P_{\alpha} P_{\beta}}{P^{2}}\right) D_{\perp}(P)\right] \equiv \\
2 M^{2} \int d \mathbf{P} f_{g}(\mathbf{P})\left[D_{\alpha \beta}(P)-\frac{P_{\alpha} P_{\beta}}{M^{2}} \beta(P)\right],
\end{array}
$$


The function $D_{\|}(P) D_{\perp}(P)$ are the same as in Eqs. (2), (3), which were found from the Fokker-Planck equation, although this equation is strictly not applicable and we derived Eqs. (21), (22) on the basis of master equation (6).

If we recalculate the time derivatives for the averaged momenta on the basis of FokkerPlanck equation (5) we would find the same form of the resulting equations $(21,22)$. Naturally, the function $f_{g}$ in both cases is different and satisfies to the relevant (Chapman-Enskog or Fokker-Planck) equations. It means that determination of the friction and diffusion coefficients by Eqs. (1)-(3) is general for the system of a two components, if the distribution function for the light component is prescribed. In this way we express the equation for the momentum of distribution function with the general velocity-dependent friction and diffusion in the velocity space. Of cause, the relation (10) is not valid anymore, nor are the Einstein relations for the velocity dependent coefficients. This consideration justifies and generalizes the statements of [3]. Interpolation relation between the friction and diffusion coefficients, which is valid for all velocities for the considered case can be written in the form:

$$
D(V)=\frac{T}{M}\left(1+\frac{2 m V^{2}}{3 T}\right) \beta(V) .
$$

which corresponds with the Einstein relation only for the case that the velocities are much smaller than thermal one $V \ll v_{T}$. In this sense a discussion of the relations between the velocity-dependent friction and diffusion coefficients on the basis of the Fokker-Planck equation [6] is relevant for non-Boltzmann collisions; for Boltzmann-type collisions this discussion seems artificial, if there is not some specific additional parameter, which makes the condition $\mathbf{q} \ll \mathbf{P}$ sufficient for using of the Fokker-Planck equation, as it is for the case of collisions in a plasma with Coulomb interaction.

At the same time in parallel with the interpolation relation (23), which, naturally, is not single-valued, the exact integral relation between the velocity dependent friction and diffusion exists as it follows from Eq. (22):

$$
\int d \mathbf{P} f_{g 0}(\mathbf{P}) D_{\alpha \gamma}(\mathbf{P})=\int d \mathbf{P} f_{g 0} \mathbf{P}_{\alpha} \mathbf{P}_{\gamma} \beta(\mathbf{P}) .
$$

This relation is the integral generalization of the Einstein's law and transfers to it for the case of velocity-independent friction and diffusion coefficients.

\section{ABSORPTION COLLISIONS}

For absorption collisions (typical, e.g., for dusty plasmas) the momentum transferred to the grain is equal to the momentum of the colliding atom (ion in the case of dusty plasmas) $\mathbf{p}$. The PT function $w(\mathbf{P}, \mathbf{q})$ in this case can be found [3] on the basis of the kinetic equation for absorption [7]

$$
w(\mathbf{P}, \mathbf{q})=f_{n}(-\mathbf{q}) \sigma_{a b s}\left(\left|\frac{\mathbf{P}}{M}+\frac{\mathbf{q}}{m}\right|\right)\left|\frac{\mathbf{P}}{M}+\frac{\mathbf{q}}{m}\right| .
$$


For the Maxwellian distribution of atoms and purely geometrical absorption $\sigma_{a b s}=\pi a^{2}$ (the particular case of the absorption cross-section for the model of dusty plasmas with the average charge of grain $Q \rightarrow 0$ ), we find for arbitrary $V$ :

$$
D(V)=2 M^{-1} T \beta(V) .
$$

For $V=0$ this result was found in [1]. Equation (26) is different from the Einstein relation because the absorption collision integral is different from the Boltzmann-type.

For the real dusty plasmas, where the average charge of grain $Q$ is very high (order of $10^{3} \div 10^{4}$ of the electron charge) the absorption cross-section can be taken in so called orbital motion limited (OML). This approach based on the assumption that there is no potential barrier for the ions moving towards the grain and the conservation of angular momentum and energy is enough to obtain the cross-section $\sigma_{a b s} \equiv \sigma_{O M L}$ of absorption for the charge $e_{\alpha}$ by grain with the radius $a$ :

$$
\sigma_{O M L}\left(Q, v_{\alpha}\right)=\pi a^{2}\left(1-\frac{2 e_{\alpha} Q}{m_{\alpha} v_{\alpha}^{2} a}\right) \theta\left(1-\frac{2 e_{\alpha} Q}{m_{\alpha} v_{\alpha}^{2} a}\right) .
$$

Here $v=\left|\mathbf{v}_{\alpha}-\mathbf{V}\right|$ is the relative velocity of the colliding particles. As it follows from Eqs. (26),(27) the Fokker-Planck equation is always applicable to the absorption collisions under consideration if $P \gg q$ due to smooth dependence of the PT function from the momentum $P$. Calculation of the velocity-dependent friction and diffusion coefficients on the basis of PT function (25) and Eqs. (1)-(3) leads to the conclusion, that friction coefficient in the model of dusty plasmas with dominant absorption collisions always negative in some velocity domain if the parameter of ion-grain interaction $\Gamma \equiv Q e_{i} / a T_{i}[2]$.For the range of parameters $\eta \equiv V^{2} / v_{T i}^{2} \ll 1$ and $1 \leq \Gamma<2$ the absorption friction coefficient for ions $\beta_{i}$ has a form:

$$
\beta_{i}(\eta, \Gamma)=2 A\left[1-\Gamma-\frac{\eta}{5}(1-3 \Gamma)\right],
$$

and negative for $\eta \leq 5(\Gamma-1) /(3 \Gamma-1)$. The coefficient $A$ is equal:

$$
A=\frac{1}{3} \sqrt{2 \pi}\left(\frac{m_{i}}{m_{g}}\right) a^{2} n_{i} v_{\mathrm{Ti}} .
$$

For $\eta \gg 1$ and arbitrary values of $\Gamma$ the equation (1) gives:

$$
\beta_{i}(\eta, \Gamma)=\frac{3}{2} A \sqrt{\frac{\pi}{\eta}}\left[1-\frac{1+2 \Gamma}{2 \eta}\right] .
$$

It means that for $\Gamma \gg 1$ and large $\eta$ the equation $\beta_{i}\left(\eta_{1} \Gamma\right)=0$ has a root $\eta_{1}(\Gamma) \simeq(1+2 \Gamma) / 2 \sim$ $\Gamma$. Naturally, for all $\Gamma>1$ there exists appropriate $\eta(\Gamma)$, which is the root of the equation $\beta_{i}(\eta, \Gamma)=0$.

Necessary to mention that it does not mean that the total friction in dusty plasmas can be negative, because there are other mechanisms of positive friction (e.e. atomic and ion scattering by grains) which provide usually totally positive friction for grains. Manifestation of negative friction due to absorption requires the very special condition. In particular we have stress that the friction coefficient $\beta_{i}$, which we discussed is finite for all values of 
velocities. Below we consider another mechanism of negative friction, for which always exist the experimental conditions.

We have to stress that Eq. (25) describes the absorption collisions without mass conservation, otherwise mass of the grains will increase and stationary solution does not exist [8][10]. In real dusty plasma both cases (stationary and non-stationary regimes) are realized, depending from the types of plasma, discharges and parameters. For the case of stationary solutions the mass conservation provides by surface recombination of ions and remove of the creating atoms back to plasma. Naturally this more complicated situation of absorption with further atom emission by grain cannot be described by PT function (25) and requires a special consideration. Therefore the mechanism of negative friction considered above has mostly illustrative interest as an example of microscopical model for negative friction.

\section{DIFFUSION IN THE COORDINATE SPACE: SELF-CONSISTENT DESCRIPTION FOR THE FOKKER-PLANCK APPROXIMATION}

Let us consider the relation of diffusion in coordinate space with diffusion in the velocity space on the basis of the Fokker-Planck equation. It means as it was shown above, that for the case of Boltzmann gas we have consider a low grain velocity (with the velocity independent friction and diffusion coefficient, when the Fokker-Planck equation is applicable). At the same time for the non-Boltzmann type of collisions, such a type as the absorption collisions in plasmas or active motion of biological objects (see [3] and the analysis below) we can consider the coefficients, which are essentially dependable from the velocity, because the Fokker-Planck equation in that case based on the processes of non-Boltzmann type. The problem of relation between the diffusion in the coordinate and velocity spaces date to Chandrasekhar [11] has been considered in a few papers (e.g. [12]- [14]) for the FokkerPlanck equation, but still is not solved self-consistently. To find the diffusion $D_{R}$ in the coordinate space we have consider a slightly inhomogeneous in space stationary solution for the Fokker-Planck equation (see inhomogeneous variant of Eq. (5):

$$
\mathbf{v} \frac{\partial f_{g}(\mathbf{P}, \mathbf{r}, t)}{\partial \mathbf{r}}=\frac{\partial}{\partial P_{i}}\left[P_{i} \beta(P) f_{g}(\mathbf{P}, \mathbf{r}, t)+M^{2} \frac{\partial}{\partial P_{j}}\left(D_{i j}(\mathbf{P}) f_{g}(\mathbf{P}, \mathbf{r}, t)\right)\right]
$$

For the distribution function one can use the expansion $f_{g}(\mathbf{p}, \mathbf{r}, t)=f_{g}^{0}(\mathbf{p}, \mathbf{r}, t)+f_{g}^{1}(\mathbf{p}, \mathbf{r}, t)$, where $f_{g}^{0}$ is has the same shape as the stationary homogeneous solution, but with coordinatedependent density $n_{g}(\mathbf{r})$ and temperature $T_{g}(\mathbf{r})$. The equation for perturbation $f_{g}^{1}$ can be written in the form:

$$
P_{i} \tilde{\beta}(P) f_{g}^{1}(\mathbf{P}, \mathbf{r}, t)+M^{2}\left(D_{i j}(\mathbf{P}) \frac{\partial}{\partial P_{j}} f_{g}^{1}(\mathbf{P}, \mathbf{r}, t)\right)=\frac{\partial}{\partial r_{j}} \Phi(P, \mathbf{r}) \equiv \Psi_{j}(P, \mathbf{r}),
$$

where the functions $\tilde{\beta}$ and $\Phi$ are equal:

$$
\begin{gathered}
\tilde{\beta}(P)=\beta(P)+\frac{s-1}{V^{2}}\left(D_{\|}(V)-D_{\perp}(V)\right)+\frac{1}{V} \frac{\partial D_{\|}(V)}{\partial V} \equiv \beta^{*}(V)+\frac{1}{V} \frac{\partial D_{\|}(V)}{\partial V}, \\
\Phi(P, \mathbf{r})=-\frac{1}{M} \int_{P}^{\infty} d P^{\prime} P^{\prime} f_{g}^{0}\left(P^{\prime}, \mathbf{r}\right) .
\end{gathered}
$$


Because for the slight inhomogeneity one can represent the function $\boldsymbol{\Psi}$ as the linear combination of the gradients:

$$
\Psi(P, \mathbf{r})=\lambda(P) \nabla n(\mathbf{r})+\gamma(P) \nabla T(\mathbf{r})
$$

it is natural to find a solution $f_{g}^{1}(\mathbf{P}, \mathbf{r}, t)$ of Eq. (32) as

$$
f_{g}^{1}(P, \mathbf{r})=\mu_{1}(P) \mathbf{P} \cdot \nabla n(\mathbf{r})+\mu_{2}(P) \mathbf{P} \cdot \nabla T(\mathbf{r}) .
$$

Taking into account that $f_{g}^{0}(P)$ is always proportional to the density of grains $n(\mathbf{r})$ we easily determine the functions $\lambda(P)$ and after that $\mu_{1}(P)$ :

$$
\begin{gathered}
\lambda(P)=-\frac{1}{M n} \int_{P}^{\infty} d P^{\prime} P^{\prime} f_{g}^{0}\left(P^{\prime}\right), \\
\mu_{1}(P)=\frac{1}{M^{2} P} \int_{0}^{P} d P^{\prime} \frac{\lambda\left(P^{\prime}\right)}{D_{\|}\left(P^{\prime}\right)} \exp \left[\int_{P}^{P^{\prime}} d P^{\prime \prime} \tau\left(P^{\prime \prime}\right)\right] .
\end{gathered}
$$

The function $\tau(P)$ under the integral in the exponent of Eq. (38) is equal:

$$
\tau(P)=\frac{P \tilde{\beta}(P)}{M^{2} D_{\|}(P)} .
$$

For self-consistent description according to Eq. (7) we can rewrite $\mu_{1}(P)$ as:

$\mu_{1}(P)=\frac{f_{g}^{0}(P)}{M^{2} P} \int_{0}^{P} d P^{\prime} \frac{\lambda\left(P^{\prime}\right)}{D_{\|}\left(P^{\prime}\right) f_{g}^{0}\left(P^{\prime}\right)} \equiv-\frac{f_{g}^{0}(P)}{M^{3} n P} \int_{0}^{P} d P^{\prime} \frac{1}{D_{\|}\left(P^{\prime}\right) f_{g}^{0}\left(P^{\prime}\right)} \int_{P^{\prime}}^{\infty} d \tilde{P} \tilde{P} f_{g}^{0}(\tilde{P})$.

The same way can be found $\mu_{2}(P)$.

Because the diffusion current is determined by the relation:

$$
\mathbf{i}_{D}=\int d \mathbf{P V} f_{g}^{1}(P, \mathbf{r}) \equiv-D_{R} \nabla \cdot n(\mathbf{r})
$$

we find straightforward in the framework of the Fokker-Planck equation the general relation:

$D_{R}=-\frac{1}{s M} \int d^{s} P P^{2} \mu_{1}(P) \equiv \frac{1}{M^{4} s n} \int d^{s} P P f_{g}^{0}(P) \int_{0}^{P} d P^{\prime} \frac{1}{D_{\|}\left(P^{\prime}\right) f_{g}^{0}\left(P^{\prime}\right)} \int_{P^{\prime}}^{\infty} d \tilde{P} \tilde{P} f_{g}^{0}(\tilde{P})$,

where $s=3$ for tree-dimensional case and $s=2$ for two-dimensional case. The values $\beta(P), D_{\|}$and $D_{\perp}$ are determined self-consistently by the relations (1),(2) and (3) via the probability transition function. Introducing the characteristic momentum $P_{0}$, velocity $V_{0}$, and constant friction $\beta_{c}$ and diffusion $D_{c}$ coefficients by formulae $P_{0}=M V_{0}, \beta(P)=\beta_{c} b(P)$ and $D(P)=D_{c} d(P)$ let us rewrite Eq.(42) in undimensional form:

$$
D_{R}=\frac{V_{0}^{4}}{s D_{c}} \int d^{s} \eta \eta \tilde{f}_{g}^{0}(\eta) \int_{0}^{\eta} d \gamma \frac{1}{d_{\|}(\gamma) \tilde{f}_{g}^{0}(\gamma)} \int_{\gamma}^{\infty} d \omega \omega \tilde{f}_{g}^{0}(\omega)
$$


where the distribution function $\tilde{f}_{g}^{0}(\eta)\left(\eta \equiv P / P_{0}\right)$ is normalized to unit:

$$
\int d^{s} \eta \tilde{f}_{g}^{0}(\eta) \equiv \int d^{s} \eta \frac{1}{d \|(\eta)} \exp \left[-\Lambda \int_{0}^{\eta} d \zeta \zeta \frac{b(\zeta)}{d_{\|}(\zeta)}\right]=1
$$

The parameter $\Lambda \equiv \beta_{c} V_{0}^{2} / D_{c}$. It means in general the relation of the diffusion coefficients in the coordinate and velocity space $(42)$ can be written as:

$$
D_{R}=\frac{V_{0}^{4}}{s D_{c}} \digamma\left(\frac{\beta_{c} V_{0}^{2}}{D_{c}}\right)
$$

where we determine the integrals in Eq. (42) as $\digamma\left(\beta_{c} V_{0}^{2} / D_{c}\right)$.

Let us consider the particular cases.

a) For the constant values of $\beta$ and $D=D_{\|}=D_{\perp}$, when the velocity dependence is absent we find:

$$
\tilde{\beta}=\beta, \tau=\frac{P \beta}{M^{2} D}, \mu_{1}(P)=\frac{1}{M^{2} D P} \int_{0}^{P} d P^{\prime} \lambda\left(P^{\prime}\right) \exp \left[\frac{\beta}{2 M^{2} D}\left(P^{\prime 2}-P^{2}\right)\right] .
$$

Let us introduce the effective temperature $T^{*}=M D / \beta$. If the stationary distribution is Maxwellian $f_{g}^{0}=f_{g 0}^{T}$ with some temperature $T$ the function $\lambda$ and $\mu_{1}$ from Eq. (46) transforms to

$$
\lambda(P)=-\frac{T f_{g 0}^{T}}{n}, \mu_{1}(P)=-\frac{T f_{g 0}^{T^{*}}}{n M^{2} D P} \sqrt{\frac{\pi}{2}} \sqrt{2 M T_{\text {eff }}} \Phi\left[\frac{P}{\sqrt{2 M T_{\text {eff }}}}\right],
$$

where $\Phi(x)$ is the error function and $1 / T_{\text {eff }} \equiv\left(1 / T-1 / T^{*}\right)$.

If the Einstein relation $M D=T \beta$ is fulfilled then $T^{*}=T$ and $T_{\text {eff }} \rightarrow \infty$. For that case we have $\mu_{1}=-T f_{g 0}^{T} / n M^{2} D$ and the diffusion coefficient in the coordinate space related with the diffusion in the velocity space in both $2 \mathrm{D}$ and $3 \mathrm{D}$ cases by the known equality:

$$
D_{R}=\frac{T^{2}}{M^{2} D}
$$

For more complicated physical problems, when the Einstein relation is not applicable, as e.g. for active particles, necessary to use the general relations derived above. In general necessary also to use the self-consistent expressions for the friction and diffusion coefficients in velocity space to find correctly the diffusion in the coordinate space, because the last one is the functional of both of them, as it follows from the general expressions (42) or (43).

However to find $\beta$ and $D$ self-consistently and determine on that basis the stationary distribution function necessary to find the probability transition function, which is known on the microscopical basis only for a restricted type of the momentum transfer processes [3] (Boltzmann-type collisions, some models of absorption, in particular in dusty plasmas, and some types of active motion). For many other cases, where the well developed microscopical model of the physical process is absent, the approximate non-self-consistent consideration, based on physical intuition, is only one way to obtain the results.

b) Let us apply now the general relations, found above, to the model of active particles (e.g. cells) capable to self-motion. The problem of active friction have been discussed 
intensively for the last decade on basis of phenomenological approach (e.g. [15]). The microscopical model of active friction for biological objects was recently suggested in [3]. Generalization of this microscopical consideration for more complicated and realistic cases, when the "driver" vector of the object, introduced in this paper, can rotate and orients on some external gradients of the ambient medium, will be considered separately. In that case the amplified force, which provides a negative friction is directed to "driver", although passive friction is antiparallel to velocity vector, and the active particle can change the direction of motion.

The PT function for the active particles has been found in [3] by considering physical processes for momentum transfer from active particles to the ambient medium by internal energy loss of the inner energy of the grain (cell). Our main finding is a "singularity" $\beta_{\varepsilon}(V) \equiv-K_{\varepsilon} / V$ in the friction coefficient for active particles and negative value of $\beta_{\varepsilon}$. Because there is also normal positive friction obliged to the surrounding medium $\beta_{0}$ the total friction is the sum $\beta_{\Sigma}=\beta_{0}+\beta_{\varepsilon}(V)$. For the simplest case $K_{\varepsilon}$ is $\mathrm{V}$-independent. For more complicated models it can be a slow function of $V$, as it was shown in [3]. We will consider here only this simplest model. The stationary distribution function for the active particles is:

$$
f_{g}(V)=C \exp \left\{-\frac{\beta_{0}}{2 D_{\Sigma}}\left(V-\frac{K_{\varepsilon}}{\beta_{0}}\right)^{2}\right\},
$$

where $D_{\Sigma}=D_{0}+D_{\varepsilon}$ is the total diffusion, which is a constant if $K_{\varepsilon}$ is $\mathrm{V}$-independent. The coefficients in Eq. (49) are found on the basis of a kinetic consideration [3]. The characteristic parameters determined above in the case under consideration can be taken $\beta_{c} \equiv \beta_{0}, V_{0}=K_{\varepsilon} / \beta_{0}, D_{c} \equiv D_{\Sigma}$ and $d(V) \equiv 1$. The physical reason for the maximum of the distribution function at $V=K_{\varepsilon} / \beta_{0}$ is evident. It is due to the balance of the normal friction and self-accelerating forces. For self-motion of grains (cells) for example in a gas of hard spheres we can use for $\beta_{0}$ and $D_{0}$ the expressions $(15,17)$. A similar $V$-dependence of the distribution function was found experimentally and phenomenologically for cells $[12,14]$. Using Eq.(43, 50) we find (for $s=3$ ):

$$
D_{R}=\frac{K_{\varepsilon}^{4}}{3 \beta_{0}^{4} D_{\Sigma}} \digamma\left(\frac{K_{\varepsilon}^{2}}{\beta_{0} D_{\Sigma}}\right)
$$

In many other cases, when the microscopic theory of active motion is not developed yet the velocity independent diffusion also seems the suitable approximation [15]. For the all these models Eq. (42) can be simplified:

$$
D_{R}=-\frac{1}{s M^{3} D} \int d^{s} P \frac{P}{f_{g}^{0}(P)} \int_{0}^{P} d P^{\prime} \lambda\left(P^{\prime}\right) f_{g}^{0}\left(P^{\prime}\right),
$$

If the characteristic constant value for the function $\beta(P)$ is $\beta_{c}$ and the characteristic momentum is $P_{0}$ (e.g. for the active particles the value of momentum, where $\beta\left(P_{0}\right)=0$ ) we can introduce the undimensional function $\phi\left(P / P_{0}\right)$ by the equality $\beta(P)=\beta_{c} \phi\left(P / P_{0}\right)$ and rewrite Eq. (42) in the form:

$$
D_{R}=-\frac{P_{0}^{4}}{s M^{4} D} \Omega(\Lambda),
$$


where the parameter $\Lambda=P_{0}^{2} \beta_{c} / M D$ and $\Omega \equiv Y / N$ is the the ratio of the integrals:

$$
\begin{array}{r}
Y=\int d^{s} \tau \tau \int_{0}^{\tau} d \eta \int_{\eta}^{\infty} d \xi \xi f_{g}^{0}(\xi) \exp \left[\Lambda \int_{\tau}^{\xi} d \omega \phi(\omega)\right] \\
N=\int d^{s} \xi f_{g}^{0}(\xi) .
\end{array}
$$

For the self-consistent scheme the distribution function itself, according to Eq. (7), is equal to:

$$
f_{g}^{0}(\xi)=C \exp \left[-\Lambda \int_{0}^{\xi} d \rho \phi(\rho)\right]
$$

\section{DIFFUSION IN THE COORDINATE SPACE ON THE BASIS OF MASTER-TYPE EQUATION}

Consideration of diffusion in the coordinate space and relation between the diffusion in coordinate and velocity space have been done in Section 3 on the basis of the Fokker-Planck kinetic equation. At the same time this approach is not enough for consideration of some special types of space diffusion, when the PT function has a specific form, in particular, posses the slow decreasing tails in the coordinate space, when the Fourier-components for such PT functions are absent. There are many very important processes, where different types of the anomalous diffusion exist [16]. This anomalous diffusion can be related not only with the coordinate, but also, in general, with time-dependent PT function. Usually the problem of anomalous diffusion is considering on the basis of fractional differentiation. This basis provides the universal description for probability distribution of grain at large distances. At the same time to describe all the distances we suggest to use the approach of PT function, when the fractional differentiation is not present at all. Naturally the results for the power-type PT functions are the same. But in general case, the PT function approach gives general and simple description for all interesting cases with a wide class of short-range behavior and a long distance tails in the coordinate space. We also formulate the timedependent equation for anomalous diffusion, which gives useful general description for many applications. These applications will be considered in details in the separate paper.

Let us consider the diffusion in the coordinate space on the basis of master equation, which describes the balance of the grains incoming and outcoming the point $r$ in the moment

$t$. The structure of this equation is formally similar to the master equation in the momentum space Eq. (6). Naturally, for the coordinate space there is no the conservation law, similar to the momentum space:

$$
\frac{d f_{g}(\mathbf{r}, t)}{d t}=\int d \mathbf{r}^{\prime}\left\{W\left(\mathbf{r}, \mathbf{r}^{\prime}\right) f_{g}\left(\mathbf{r}^{\prime}, \mathbf{t}\right)-W\left(\mathbf{r}^{\prime}, \mathbf{r}\right) f_{g}(\mathbf{r}, t)\right\}
$$

The probability transition $W\left(\mathbf{r}, \mathbf{r}^{\prime}\right)$ describes probability for a grain to transfer from the point $\mathbf{r}^{\prime}$ to the point $\mathbf{r}$ per unit time. We can rewrite this equation in the coordinates $\rho=\mathbf{r}^{\prime}-\mathbf{r}$ and $\mathbf{r}$ as: 


$$
\frac{d f_{g}(\mathbf{r}, t)}{d t}=\int d \rho\left\{W(\rho, \mathbf{r}+\rho) f_{g}(\mathbf{r}+\rho, \mathbf{t})-W(\rho, \mathbf{r}) f_{g}(\mathbf{r}, t)\right\} .
$$

If we suggest that the characteristic displacements are small and expand Eq. (57) we arrive to the "Fokker-Planck" form of the equation for the density distribution $f_{g}(\mathbf{r}, t)$

$$
\frac{d f_{g}(\mathbf{r}, t)}{d t}=\frac{\partial}{\partial r_{\alpha}}\left[A_{\alpha}(\mathbf{r}) f_{g}(\mathbf{r}, t)+\frac{\partial}{\partial r_{\beta}}\left(B_{\alpha \beta}(\mathbf{r}) f_{g}(\mathbf{r}, t)\right)\right]
$$

The coefficients $A_{\alpha}$ and $B_{\alpha \beta}$ describe the acting force and diffusion, respectively, can be written as the functionals of the probability function (PT) in the coordinate space $W$ in the form:

$$
A_{\alpha}(\mathbf{r})=\int d^{s} \rho \rho_{\alpha} W(\rho, \mathbf{r})
$$

and

$$
B_{\alpha \beta}(\mathbf{r})=\frac{1}{2} \int d^{s} \rho \rho_{\alpha} \rho_{\beta} W(\rho, \mathbf{r})
$$

For the isotropic case the the probability function depends from $\mathbf{r}$ the modulus of $\rho$. For homogeneous medium, when $r$-dependence of the PT is absent the coefficients $A_{\alpha}=0$ and the diffusion coefficient is constant $B_{\alpha \beta}=\delta_{\alpha \beta} B$, where B is the integral

$$
B=\frac{1}{2 s} \int d^{s} \rho \rho^{2} W(\rho)
$$

This consideration cannot be applied to the specific situations, when the integral in Eq. (61) is infinite. In that case we have investigate the general transport equation (56). We will consider the problem for homogeneous and isotropic case, when PT function depends only from modulus $|\rho|$. By Fourier-transformation we arrive to the form of Eq. (56):

$$
\frac{d f_{g}(\mathbf{k}, t)}{d t}=\int d^{s} \rho[\exp (i \mathbf{k} \rho)-1] W(|\rho|) f_{g}(\mathbf{k}, t) \equiv X(\mathbf{k}) f_{g}(\mathbf{k}, t),
$$

where $X(\mathbf{k}) \equiv X(k)$. Let us consider the simple form of PT function with a power dependence from distance $W(\rho)=C /|\rho|^{\alpha}$, where $C$ is a constant. For one-dimensional case we find:

$$
X(k) \equiv-4 \int_{0}^{\infty} d \rho \sin ^{2}\left(\frac{k \rho}{2}\right) W(\rho)=-2^{3-\alpha} C|k|^{\alpha-1} \int_{0}^{\infty} d \zeta \frac{\sin ^{2} \zeta}{\zeta^{\alpha}}
$$

For the values $1<\alpha<3$ this function is finite and equal

$$
X(k)=-2 C \Gamma(1-\alpha)|k|^{\alpha-1} \cos \frac{(1-\alpha) \pi}{2},
$$

where $\Gamma$ is the Gamma-function. At the same time the integral (61) for such type of PT functions is infinite, because usual diffusion is absent. The considered procedure for the simplest cases of power dependence of $\mathrm{PT}$ function is equivalent to the equation with fractional space differentiation [17-19]: 


$$
\frac{d f_{g}(x, t)}{d t}=C \Delta^{\mu / 2} f_{g}(x, t)
$$

where $\Delta^{\mu / 2}$ is a fractional Laplacian, a linear operator, whose action on the function $f(x)$ in Fourier space is described by $\Delta^{\mu / 2} f(x)=-\left(k^{2}\right)^{\mu / 2} f(k)=-|k|^{\mu} f(k)$. In the considered above case $\mu \equiv(\alpha-1)$, where $0<\mu<2$. At the same time for more general PT functions, which are (for arbitrary values $\rho$ ) are not proportional to the $-\alpha$ th power of $\rho$, the method described above is also applicable, although the fractional derivative is not exists.

For the cases of purely power dependence of PT the non-stationary solution for density distribution describes so-called super-diffusion (or Levy flights). The solution of Eq. (65) in Fourier space reads:

$$
f_{g}(k, t)=\exp \left(-C|k|^{\mu} t\right)
$$

which in coordinate space corresponds to a so-called symmetric Levy stable distribution:

$$
f_{g}(x, t)=\frac{1}{(k t)^{1 / \mu}} L\left[\frac{x}{(k t)^{1 / \mu}} ; \mu, 0\right]
$$

For general case as it follows from Eq. (62)

$$
f_{g}(k, t)=C_{1} \exp [X(k) t]
$$

with some constant $C_{1}$.

Let us turn to the three-dimensional case for the PT function equal $W=C /|\rho|^{\alpha}$ with a constant $C>0$. Then the function $X(k)$ is equal

$$
X(k) \equiv 4 \pi \int_{0}^{\infty} d \rho \rho^{2}\left(\frac{\sin (k \rho)}{k \rho}-1\right) W(\rho)=4 \pi C k^{\alpha-3} \int_{0}^{\infty} d \zeta \frac{1}{\zeta^{\alpha-2}}\left(\frac{\sin \zeta}{\zeta}-1\right)
$$

This integral is finite for the values $3<\alpha<5$. If we return to the variable $\mu \equiv \alpha-s$ ( $\mathrm{s}=3$ for 3-dimensional case and equal to the power of $k$ in the function $X(k)$ ) we find the same limitation as in one-dimensional case $0<\mu<2$, which characterizes the fractional derivative.

Naturally, consideration on the basis of PT function given above, permits to avoid the fractional differentiation method and to consider more general physical situations of the non-power probability transitions. Let us consider that for a simple example. Taking (for one-dimensional case) the PT function $W(\rho)$ in the form

$$
W(\rho)=C \frac{1-\exp \left[-\sigma \rho^{p}\right]}{\rho^{\alpha}}
$$

with $p>0$, we arrive (e.g. for one-dimensional case) to the function $X(k)$ :

$$
X(k)=-2^{3-\alpha} C|k|^{\alpha-1} \int_{0}^{\infty} d \zeta \frac{\left\{1-\exp \left[-\sigma(2 \zeta /|k|)^{p}\right]\right\} \sin ^{2} \zeta}{\zeta^{\alpha}} \equiv-2^{3-\alpha} C|k|^{\alpha-1} Y\left(\sigma /|k|^{p}, \alpha\right)
$$


As easy to see the function $Y\left(\sigma /|k|^{p}, \alpha\right)$ is finite for $1<\alpha<p+3$, because for the small values of distances for $p>0$ divergence is suppressed also for some powers $\alpha>3$. Simple calculation for $\alpha=2$ and $p=1$ leads to the result, which cannot be found by the fractional differentiation method

$$
Y(\sigma /|k|, 2)=\frac{\pi}{2}-\arctan (|k| / \sigma)+\frac{\sigma}{2|k|} \ln \left[1+k^{2} / \sigma^{2}\right] .
$$

The asymptotic behavior of the function $X(k)$ for $k \rightarrow \infty$ is similar, as it follows from Eq. (72), to the case $W(x)=c / \rho^{\alpha}$. Therefore the universal behavior of $X(k)$ is provided by asymptotical properties of PT function for $1<\alpha<3$.

For the normal Gaussian diffusion (see e.g. Eqs. (58), (61)) for arbitrary $s$ the function is equal $X(k)=-B k^{2}$. It leads to the Gaussian distribution in the coordinate space and to the usual time dependence of the square-mean displacement $\left\langle r^{2}\right\rangle \sim B t$. For different types of anomalous diffusion this relation is violated and the dependence $\left\langle r^{2}(t)\right\rangle$ has to be calculated on the basis of concrete anomalous distribution, which is determined by the respective equation for $f_{g}(\mathbf{r}, t)$. As we already demonstrated and will show below the crucial function, which determines the process is the PT function $W$.

We also would like to mention in the connection of the problem of generalized description of diffusion that the time dependence of the density distribution for the time dependent PT function can be very different from the classical diffusion. This kind of problems relates to the class of the stochastic transport, which is very popular in modern physics and describes so called subdiffusive behavior [20], e.g. photoconductivity in strongly disordered and glassy semiconductors or the resonance radiative transfer in a plasma [21], which posses many other applications. Here we only shortly formulate the problem, leaving it for the detail consideration in the next paper. For this purpose we formulate a more general transport equation for density distribution:

$$
f_{g}(\mathbf{r}, t)=f_{g}(\mathbf{r}, t=0)+\int_{0}^{t} d \tau \int d \mathbf{r}^{\prime}\left\{W\left(\mathbf{r}, \mathbf{r}^{\prime}, \tau, t-\tau\right) f_{g}\left(\mathbf{r}^{\prime}, \tau\right)-W\left(\mathbf{r}^{\prime}, \mathbf{r}, \tau, t-\tau\right) f_{g}(\mathbf{r}, \tau)\right\} .
$$

For the case of stationary PT function we return to Eq. (56). If there is no memory, but PT function is a function of current time $\tau$ we arrive to the equation more general than Eq. (56), which describes the density evolution, with prescribed time dependence of PT. In particular in that case for slow space dependence of the function $W$ we find the equation for diffusion similar to Eq. (58)-(60) with time-dependent coefficients $A_{\alpha}(\mathbf{r}, t)$ and $B_{\alpha \beta}(\mathbf{r}, t)$, which are calculated on the basis of the PT function $W(\rho, \mathbf{r}, t)$.

If the system posses memory, then, in the simplest case, when the function $W$ can be expanded in spirit of Fokker-Planck approximation in the coordinate space we arrive to the following form of Eq. (73)

$$
f_{g}(\mathbf{r}, t)=f_{g}(\mathbf{r}, t=0)+\int_{0}^{t} d \tau \frac{\partial}{\partial r_{\alpha}}\left[A_{\alpha}(\mathbf{r}, t-\tau) f_{g}(\mathbf{r}, \tau)+\frac{\partial}{\partial r_{\beta}}\left(B_{\alpha \beta}(\mathbf{r}, t-\tau) f_{g}(\mathbf{r}, \tau)\right)\right] .
$$

If the function $W$ posses memory and depends only from the difference $t-\tau$, but cannot be expanded in the coordinate space as in the case (74) we can use the Laplacetransformation in time to find: 


$$
f_{g}(\mathbf{r}, z)=\frac{f_{g}(\mathbf{r}, t=0)}{z}+\int d \mathbf{r}^{\prime}\left\{W\left(\mathbf{r}, \mathbf{r}^{\prime}, z\right) f_{g}\left(\mathbf{r}^{\prime}, z\right)-W\left(\mathbf{r}^{\prime}, \mathbf{r}, z\right) f_{g}(\mathbf{r}, z)\right\} .
$$

To arrive to this equation we suggested that due to fast convergence the upper limit $t$ in the integral $\int_{0}^{t} d \tau W(\tau) \exp (-z \tau)$ with $\operatorname{Im} z>0$ can be changed on infinity and determine $W\left(\mathbf{r}^{\prime}, \mathbf{r}, z\right)$ by the equality:

$$
W\left(\mathbf{r}^{\prime}, \mathbf{r}, z\right)=\int_{0}^{\infty} d \tau W\left(\mathbf{r}^{\prime}, \mathbf{r}, \tau\right) \exp (-z \tau)
$$

(The same approach can be used to simplify the Fokker-Planck-type equation (74)). For the space homogeneous case Eq. (75) can be Forier-transformed and rewritten in the $k, z$ variables:

$$
f_{g}(\mathbf{k}, z)=\frac{f_{g}(\mathbf{k}, t=0)}{z[1-X(\mathbf{k}, z)]}
$$

where

$$
X(\mathbf{k}, z)=\int d^{s} \rho[\exp (i \mathbf{k} \rho)-1] W(|\rho|, z)
$$

If the PT function is time-independent $W(|\rho|, z)=W(|\rho|) / z$ and $f_{g}(\mathbf{k}, t=0)=$ const. we return, as easy to see, to the case of abnormal diffusion considered above in Eqs. (62)-(72). For a generale multiplicative form of PT function $W(|\rho|, \tau)=W_{1}(|\rho|) W_{2}(\tau)$ the function $X(k, z)$ is the product:

$$
X(\mathbf{k}, z) \equiv X_{1}(k) X_{2}(z)=\int d^{s} \rho[\exp (i \mathbf{k} \rho)-1] W_{1}(|\rho|) \cdot \int_{0}^{\infty} d \tau W_{2}(\tau) \exp (-z \tau) .
$$

For the power $\tau$-dependence $W_{2}=C / \tau^{\gamma}$ with $\gamma<1$ the integral $X_{2}(z)$ is equal

$$
X_{2}(z)=\frac{1}{z^{1-\gamma}} \Gamma(1-\gamma)
$$

The distribution function $(77)$ in $k, z$ space in that case reads

$$
f_{g}(\mathbf{k}, z)=\frac{f_{g}(\mathbf{k}, t=0)}{\left[z-z^{\gamma} \Gamma(1-\gamma) X_{1}(k)\right]}
$$

The detail consequences and physical applications of more complicated dependence $W(|\rho|, z)$ from $z$ will be considered in the separate paper.

\section{GENERALIZED FRICTION}

Let us consider now the generalized expression for the friction force $\mathbf{F}_{f}$ on the basis of the Fokker-Planck equation for charged grains. As it was shown in [3] for scattering of the ion stream with velocity $\mathbf{u}$ by moving grain with momentum $\mathbf{P}_{\mathbf{0}}$ the of $\mathrm{PT}$ function $\tilde{w}_{s}\left(\mathbf{P}_{\mathbf{0}}, \mathbf{y}, \mathbf{q}\right)$ satisfies the equality: 


$$
\tilde{w}_{s}\left(\mathbf{P}_{\mathbf{0}}, \mathbf{y}, \mathbf{q}\right)=w_{s}\left(\mathbf{P}_{\mathbf{0}}-\mathbf{y}, \mathbf{q}\right),
$$

where $w_{s}(\mathbf{P}, \mathbf{q})$ is the PT for scattering of an isotropic system of ions by moving grains and $\mathbf{y}=M \mathbf{u}$. The generalized friction force for one grain can be represented through the friction coefficient:

$$
\mathbf{F}_{f s}\left(\mathbf{P}_{\mathbf{0}}-\mathbf{y}\right)=-\left(\mathbf{P}_{\mathbf{0}}-\mathbf{y}\right) \beta_{s}\left(\left|\mathbf{P}_{\mathbf{0}}-\mathbf{y}\right|\right) .
$$

In particular, Eq. (83) describes both limiting cases: the friction force itself (for $\mathbf{u}=0$ ) and the drag force itself (for $\mathbf{P}_{0}=0$ ), acting on the grain due to ion scattering with a non-zero driven velocity. For two limiting cases of the friction force itself $\mathbf{F}_{f 0 s}$ for the grain with momentum $\mathbf{G}$ and immobile ions and for the opposite case - ion drag itself $\mathbf{D}_{i s}$ with the ion velocity $\mathbf{u}=\mathbf{G} / M$ there is natural relation $\mathbf{F}_{f 0 s}(\mathbf{G})=-\mathbf{D}_{i s}(\mathbf{G})$. This picture leads to the (for practical applications important) generalization for a few species of the lighter particles, which we consider below. The particular result for $\mathbf{D}_{i s}$ for the case $|\mathbf{u}| \ll v_{T_{i}}$ and the Coulomb scattering cross-section can be written in the form:

$$
\mathbf{D}_{i s}=2 A_{0} M \mathbf{u} \Gamma^{2} \ln \Lambda
$$

where $A_{0}=\frac{\sqrt{2 \pi}}{3}\left(m_{i} / M\right) a^{2} n_{i} v_{T_{i}}, v_{T_{i}} \equiv \sqrt{T_{i} / m_{i}}$, and $a$ is the radius of grain. For dusty plasmas usually the parameter $\Gamma \equiv e^{2} Z_{g} Z_{i} / a T_{i} \gg 1$, and the Landau logarithm $\ln \Lambda$ is given in $[22],[23]$.

For the collecting PT function $\tilde{w}_{c}(\mathbf{P}, \mathbf{y}, \mathbf{q})$, based on the existing collecting collision integral [7], a representation similar to Eq. (82) is not valid [3]. Instead of Eq. (82) we have:

$$
\tilde{w}_{c}(\mathbf{P}, \mathbf{y}, \mathbf{q})=w_{c}(\mathbf{P}-\mathbf{y}, \mathbf{q}+m \mathbf{u}),
$$

where $w_{c}(\mathbf{P}, \mathbf{q})$ is the PT for the collecting process (in the case under consideration for the ions) in the isotropic ion system $(\mathbf{u}=0)$, e.g., the Maxwellian one. The collecting part of the ion friction force for $\eta \equiv v^{2} / 2 v_{\mathrm{Ti}}^{2} \ll 1$ and arbitrary $\Gamma$ has a form [2], [3]:

$$
\mathbf{F}_{f c}=-M \mathbf{V} \beta(\eta, \Gamma)=-2 M \mathbf{V} A_{0}\left[1-\Gamma-\frac{\eta}{5}(1-3 \Gamma)\right] .
$$

For $\Gamma>1$ this formula describes a negative collecting friction $\mathbf{F}_{f c}$ parallel to $\mathbf{V}$. At the same time the collecting ion drag force $\mathbf{D}_{i c}$ (derived in [22] and rigorously justified later in [3]) for $\xi \equiv u^{2} / 2 v_{\mathrm{Ti}}^{2} \ll 1$ and arbitrary $\Gamma$ can be written as:

$$
\mathbf{D}_{I c}=8 A_{0} M \mathbf{u}\left[1+\frac{\Gamma}{2}\right] \text {. }
$$

The difference of the modulus of forces in Eqs. (86) and (87) (for the case $u=V$ ) is the reflection of the collecting PT property, expressed by Eq. (85) and the structure of the collision collecting integral in dusty plasmas, when all the ions which cross the grain surface are absorbed and therefore completely transfer their momentum to the grain, although the mass transfer is considered as negligible. The problem of the mass transfer has been considered in [?]- [?] where a mass kinetic variable for dusty plasmas was introduced. The explicit solutions obtained in these papers were non-stationary. For RF dusty plasmas we are interested to find stationary solutions for the grain distribution function. These solutions take into account the surface recombination of the ions that bombarded the grain and remove the created atoms to ambient plasmas. Such a kinetic model (very close to many real experiments in RF discharge with dust) is in the process of being developed. 


\section{NEGATIVE GENERALIZED FRICTION FOR THE CASE OF ION FLOW}

Let us consider now the important generalization of the above approach for the case of two species of light particles, which transfer momentum to the grains. We assume an ion stream with velocity $\mathbf{u}$, which can be scattered and absorbed by the grains (with velocity $\mathbf{V}$ ) and an atomic subsystem with an isotropic velocity distribution function, e.g., a Maxwellian distribution. Then by using the method developed in [3] and assuming that for the considering model the equality (82) is satisfied, we arrive at the total force acting on the grains as expressed through the total friction coefficient $\beta_{t}(V)$ :

$$
\begin{gathered}
\mathbf{A}_{\alpha}(P, y)=\mathbf{P}_{\alpha} \beta_{t}(V), \\
\beta_{t}(V)=\beta_{a}(V)-\frac{y}{P} \beta_{i}(y) .
\end{gathered}
$$

Using the approximations for atomic and ion friction coefficients $\beta_{a}(V) \simeq \beta_{a}(0)$, where

$\beta_{a}(0)=8 \frac{\sqrt{2 \pi}}{3}\left(m_{a} / M\right) a^{2} n_{a} v_{T_{a}}$ (scattering of a point atom on the hard sphere grain) and $\beta_{i}(V) \simeq 2 A_{0} \Gamma^{2} \ln \Lambda$. This approximation for $\beta_{i}(V)$ is valid for dominant ion scattering drag [24] and permits to avoid the problem of possible negative friction related with the mechanism of ion absorption, discussed in [2]. Such a way we arrive, for the simplest case, when the vectors of $\mathbf{P}$ and $\mathbf{u}$ are parallel, at the picture typical for negative effective total friction. The total friction coefficient

$$
\beta_{t}(V)=\beta_{a}(0)\left[1-\frac{u m_{i} n_{i} v_{T_{i}}}{2 V m_{a} n_{a} v_{T_{a}}} \Gamma^{2} \ln \Lambda\right]
$$

becomes negative for small grain velocities.

\section{CONCLUSIONS}

The paper devoted to consideration velocity-dependent friction and diffusion coefficients in the velocity space and diffusion, including anomalous one, in the coordinate space. We established the general relations, which determine these coefficients (including tensorial structure of diffusion) and showed, that this relations exist not only for the Fokker-Planck equation, which applicability is restricted, at least for the Boltzmann-type of collisions, by low range of grain velocity. On the basis of master equation with the prescribed PT function we showed validity of these general relations for arbitrary grain velocities.

We also considered the application of the PT function method to the ion absorption collisions in dusty plasmas, where due to the specific structure of the absorption cross-section and PT function the negative friction appears for a domain of low grain velocity. Necessary to mention that this result is valid for the considered model [2], but for application of the results to the real dusty plasmas, where the additional processes of surface ion recombination and atom remove to the plasma are exist, the elaborated models have to be developed.

The important question about relation between the diffusion coefficients in the velocity and coordinate spaces is solved for the Fokker-Planck equation. For open systems, or for the 
systems with velocity dependent coefficients the relation with coordinate diffusion is essentially more complicated, than in the usual case of time independent coefficients, connected by the Einstein relation.

The problems of anomalous diffusion are considered on the basis of PT for the mastertype equation in the coordinate space. Consideration presented above permits to avoid the method of fractional space differentiation (coinciding with the results obtained by this method in the particular cases of power-type dependencies of PT) and to extend the results for a wide class of PT functions. The extension of this approach is used to formulate the general integral equation for distribution function in the coordinate space, applicable to description of very different types of time-dependent normal and anomalous diffusion. This results, as we trust, can be important for very different applications.

In many cases the PT function in the velocity space depends from some other vectors, except grain velocity. It can be ion driven velocity, for example, in dusty plasmas, or "driver" vector of cells in biological systems [3], or some additional vector for colliding inelastic grains etc. For these cases we have consider the methods of PT function determination and calculation the generalized friction and diffusion. Some development of these approach is also presented in the paper in application to scattering and absorption of ions by grains in dusty plasmas.

For the case of scattering of ions, which have some driven velocity, by grains, moving in the slightly ionized dusty plasmas with essential friction between grains and atomic component we show manifestation of negative friction for low grain velocities. This process can be easily realized for some parameters of dusty plasma, providing acceleration and heating of grains.

\section{ACKNOWLEDGMENTS}

Authors thanks W. Ebeling, A.M.Ignatov and I.M. Sokolov for valuable discussions of some problems, reflected in this work. 


\section{REFERENCES}

[1] P.P.J.M. Schram, A.G. Sitenko, S.A. Trigger, A.G. Zagorodny, Phys. Rev. E63, 016403 (2000).

[2] S.A.Trigger, $\quad$ A.G.Zagorodny, $\quad$ Contr. $\quad$ Pl. $\quad$ Phys. $43 \quad 381 \quad$ (2003); http://arXiv.org/abs/physics/0211013.

[3] S.A.Trigger, Phys. Rev. E 67, 046403 (2003).

[4] P.Resibois, M. De Lenner. Classical Kinetic Theory of Fluids, John Wiley and Sons, N.-Y., 1977.

[5] E.M.Lifshitz, L.P.Pitaevskii. Physical Kinetics, Course of Theoretical Physics V.X, Publishing "Science", Moscow, 1979.

[6] Yu.L.Klimontovich.Statistical Theory of Open Systems I, Kluwer Academic Publishers, Dordrecht, 1995.

[7] A.M.Ignatov, Plasma Phys. Rep. 24, 677 (1998).

[8] S.A. Trigger, Contrib. Plasma Phys. 41, 331 (2001).

[9] A.M. Ignatov, S.A. Trigger, W. Ebeling, and P.P.J.M. Schram, Phys. Lett. A293, 141 (2001).

[10] A.M. Ignatov, S.A. Trigger, S.A.Maiorov, W. Ebeling, Phys.Rev. E65, 046413 (2002).

[11] S. Chandrasekhar, Rev. Mod. Phys. 15, 1 (1943).

[12] M. Scheinbein, and H. Gruler, Bull. Math. Biology 55, 585 (1993).

[13] A.S. Mikhailov, and D. Meinköhn, In: Stochastic Dynamics, edited by L. SchimanskyGeier, T. Pöschel, vol. 484 of Lectures Notes in Physics, (Springer, Berlin), 1997, p. 334.

[14] M. Schienbein, K. Franke, and H. Gruler, Phys.Rev E. 49, 5462 (1994).

[15] U. Erdmann, W. Ebeling, L. Schimansky-Geier, and F. Schweitzer, Eur. Phys. J. B15, $105(2000)$.

[16] R.Metzler, and J.Klafter, Phys. Rep. 339, 1 (2000).

[17] V.Seshadri, Phys. Rev. A 36, 892 (1987).

[18] H.C.Fogedby, Phys. Rev. Lett. 73, 2517 (1994).

[19] D.Brockmann, and I.M.Sokolov, Chemical Physics 284, 409 (2002).

[20] H.Scher, and E.W.Montroll, Phys. Rev. B 12, 2455 (1975).

[21] V.Yu. Zaburdaev, and K.V.Chukbar, Journal of Experimental and Theoretical Physics, 94, $252(2002)$.

[22] S.A. Khrapak, A.V. Ivlev, G.E. Morfill, and H.M. Thomas., Phys. Rev. E 66, 046414 (2002).

[23] P.P.J.M. Schram, S.A. Trigger, and A.G. Zagorodny, New J. Phys. 5, 27 (2003).

[24] S.A. Trigger, W. Ebeling, A.M. Ignatov, and I.M. Tkachenko, Contr. Pl. Phys. 43, 377 (2003). 